\title{
REVIEW
}

\section{Use of bacille Calmette-Guérin in superficial bladder cancer}

\author{
J-P Meyer, R Persad, D A Gillatt
}

Postgrad Med J 2002;78:449-454

Intravesical bacille Calmette-Guérin (BCG) has been used by urologists for several years after its first reported use as a cancer therapy in the 1930s. Morales in 1976 described the usage of BCG as a once weekly intravesical instillation for six weeks; this is a treatment regimen that still exists today. Its success as a treatment depends on it being used appropriately. It is employed: (1) to treat carcinoma in situ or occasionally residual papillary tumours; (2) to reduce the number and frequency of recurrent high grade superficial tumours; and (3) to prevent disease progression (although this remains a controversial point, on which there is no consensus view). Unfortunately, the more widespread use of BCG is often limited due its high side effect profile. Present research is directed towards reducing its side effect profile, improving its efficacy, and understanding its exact mechanism of action, which is not fully understood.

See end of article for authors' affiliations

Correspondence to: Mr Jon-Paul Meyer, Bristol Urological Institute,

Southmead Hospital,

Westbury-on-Trym, Bristol

BS10 5NB, UK;

ipmeyer@doctors.org.uk

Submitted

8 January 2002

Accepted 22 April 2002
$\mathrm{E}$ ach year in England and Wales, around 8500 men and 3000 women develop cancer of the bladder. Bladder cancer is the fourth most common cancer in men and the eighth most common in women. In the developed world, transitional cell carcinoma (rather than squamous cell carcinoma or adenocarcinoma) is responsible for most bladder carcinoma. Bladder cancer can be divided into superficial and muscle invasive cancer. Superficial bladder cancer involves the bladder wall no deeper than the subepithelial tissue and has not reached the detrusor muscle. Of patients presenting with transitional cell carcinomas, $70 \%$ have superficial tumours (50\% pTa and $20 \%$ pTl) not invading the detrusor muscle, and $25 \%$ have muscle invasive tumours, the remaining

\section{Box 1: Prognostic factors for recurrence}

- Number of tumours; more tumours mean more frequent recurrences.

- Previous recurrence rate* or a recurrence at three months.

- Size of the tumour; those $>3 \mathrm{~cm}$ carry a worse prognosis.

- Grade of anaplasia. ${ }^{2}$

- Presence of CIS.

*The recurrence rate being the number of positive cystoscopies per year over the number of cystoscopies performed per year.
$5 \%$ being carcinoma in situ (CIS); see fig 1 . Of those with newly diagnosed superficial bladder tumours, approximately $30 \%$ are multifocal at presentation, $60 \%-70 \%$ will recur, and $10 \%-20 \%$ will undergo stage progression to muscle invasive or metastatic disease.

\section{TREATMENT OF SUPERFICIAL BLADDER CANCER}

The treatment of bladder cancer in general depends on numerous factors, including stage and grade of the disease, patient age, and their co-morbidity. The standard treatment of superficial bladder cancer is a transurethral resection, this initial resection usually removes the tumour but it is known that they have a great propensity to recur. It is important to realise that some tumours progress, and in those which do it is vital that they are recognised so that further treatment can be instigated. A significant breakthrough in the last decade has been the clear definition of prognostic factors, which determine later recurrence. Using these factors it is possible to identify different patient subgroups and tailor their treatment and subsequent follow up accordingly. The most important factors are easily assessed at transurethral resection and are listed in box 1 .

For progression, tumour grade is the most important factor. It is also known that Tl tumours have a worse prognosis than Ta tumours. Using these factors patients with superficial bladder cancer can be divided into three groups: (1) the lowest risk group with a single less than $3 \mathrm{~cm}$ Ta Gl tumour, and a recurrence rate of less than one tumour per year; (2) a high risk tumour of Tl G3, multifocal, large, highly recurrent, and CIS; and (3) other tumours of intermediate risk. Recent studies further confirm these criteria..$^{3-5}$

\section{BACILLE CALMETTE-GUÉRIN (BCG)}

Urologists have for a number of years employed intravesical therapies in order to reduce the number and rate of recurrences of superficial bladder cancer, or to prevent them all together. One such therapy is BCG immunotherapy.

\section{DEVELOPMENT OF BCG}

In 1904, Novard isolated Mycobacterium bovis from a cow with tuberculous mastitis. During the passage through the laboratory that followed, the

Abbreviations: BAK, BCG activated killer cells; BCG, bacille Calmette-Guérin; CIS, carcinoma in situ; EORTC, European Organisation for the Research and Treatment of Cancer; ICAM, intracellular adhesion molecule; MHC, major histocompatibility complex; SWOG, Southwest Oncology Group 


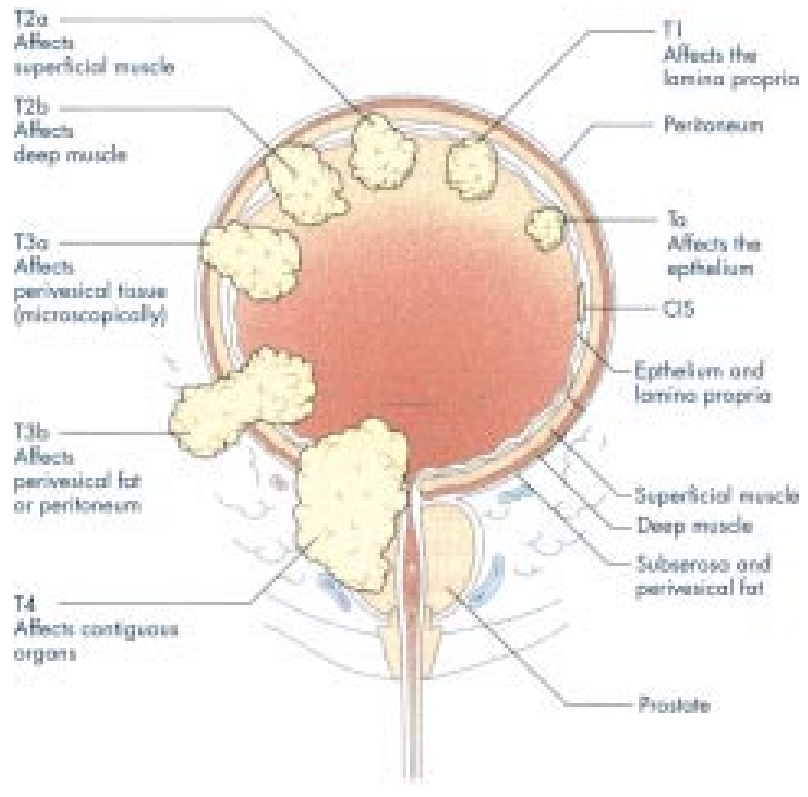

Figure 1 Staging of bladder cancer.

mycobacterium culture showed a strong tendency to clump. In order to prevent adhesion, in 1908 Calmette and Guérin added bovine bile to the culture medium. In this culture, the $M$ bovis showed a loss of virulence for animals in the many passages that took place over the following years, until by 1920 the culture was regarded as being avirulent. It took 231 passages through the laboratory over a period of 13 years to achieve this result. ${ }^{6}$ This special $M$ bovis strain was named Bacillus of Calmette and Guérin (Bacille de Calmette et Guérin, BCG).

\section{HISTORY OF USAGE OF BCG}

Tuberculosis was noted to have an antitumour effect at the start of the 20th century. It was Pearl, who in an autopsy series at the Johns Hopkins Hospital in 1929 reported a lower frequency of cancer in patients with tuberculosis. ${ }^{7}$ In the 1930s the use of BCG as a cancer therapy was first raised, but enthusiasm for this did not mount until the 1950s and 1960s. In 1969, Mathe et al produced a paper on the treatment of lymphoblastoid leukaemia with BCG, ${ }^{8}$ which produced promising results, but unfortunately others could not reproduce their results. This coupled with the development of successful modern chemotherapy and radiotherapy meant enthusiasm for BCG as a treatment waned. Further research carried out by Coe and Feldman rekindled interest in BCG with the demonstration of a strong delayed hypersensitivity reaction to BCG in the guinea pig bladder.9 This knowledge aided Morales et al who in 1976 carried out the first clinical trial with intravesical BCG. ${ }^{10}$ They were able to demonstrate a remarkable decrease in the rates of recurrence of superficial bladder cancer in nine patients. A randomised prospective trial by Lamm et al in 1980 confirmed these earlier observations. ${ }^{11}$ Since this time it has been shown that BCG when used has inferred benefit to those with superficial bladder cancer in terms of decreased recurrence rate and increased time to recurrence.

\section{DIFFERENT STRAINS OF BCG}

There are several different strains of BCG available. The more commonly used strains include the Tice strain produced by Organon Teknika, the RIVM strain (Rijksinstituut voor Volksgezonheid en Milieuhhygiene) produced by Bilthoven, and the Connaught strain produced by Connaught Laboratories. Morales used a strain of BCG which came from the Institut Armand Frappier in Montreal, in fact BCG-Connaught is derived from this strain.

\section{Box 2: Uses of BCG immunotherapy}

- To treat CIS or occasionally residual papillary tumours.

- To reduce the number and frequency of recurrent tumours.

- To prevent disease progression, although this remains a controversial point.

As there are several commercially available strains of BCG, uncertainty exists in the literature about whether the various strains of BCG have identical immunotherapeutic efficacy and identical clinical effectiveness. Given this fact direct comparison between clinical studies is difficult when different strains of BCG have been used.

\section{TREATMENT REGIMEN}

This consists of a once weekly instillation of BCG for six weeks as described by Morales. Patients are given live attenuated BCG mixed in $50 \mathrm{ml}$ of normal saline instilled into the bladder via a urethral catheter. Before instillation urinary tract infection is excluded and the catheter is introduced in an atraumatic way. The patient retains the fluid within the bladder for an hour and during this period spends 15 minutes prone, 15 minutes supine, and 15 minutes on each side. This is to ensure that all the bladder mucosa comes into contact with the BCG.

There is a small risk of tuberculosis infection, therefore the staff administering the BCG are suitably protected with masks, goggles, gloves, and gowns. Patients are advised to bleach their toilets after urinating, so as to neutralise any BCG that may be remaining in the urine.

After the conclusion of the six week course of BCG the patient undergoes a cystoscopy. If the bladder is free of tumour recurrence, then the patient is entered into a programme of regular cystoscopic follow up. If tumour is seen to have recurred then the patient can after resection have a further course of BCG. A second six week course of BCG will improve the tumour free rate by $20 \%-30 \%{ }^{12}{ }^{13}$; this means that those receiving a second course of BCG have a $20 \%-30 \%$ higher chance of being tumour-free than those who received a single course of BCG. However, it is important to consider that patients failing more than two courses of BCG have only a $20 \%$ chance of being tumour-free and a 50\% risk of metastatic bladder cancer. ${ }^{13}$

Some centres give further courses of BCG as maintenance therapy, in an attempt to maintain the bladder's immunity to further tumours. The crucial question as to whether or not this actually provides continuous protection was open to debate until Lamm et al published their findings. ${ }^{14}$ In Lamm's study patients in the maintenance arm received BCG instillations once a week for three weeks at 3, 6, 12, 18, 24, 30, and 36 months after the initial course of BCG. There was an estimated 35.6 month median recurrence free survival in those not receiving maintenance therapy compared to 76.8 months in those receiving maintenance therapy $(\mathrm{p}<0.001)$. At present, this schedule is the best that can be advocated for those patients at high risk of both recurrence and progression. It should be noted, however, that only small numbers of patients actually complete the full course of maintenance therapy, as a result of the increased number of side effects experienced due to the toxicity associated with multiple doses of intravesical BCG.

To further investigate the question of maintenance therapy the European Organisation for the Research and Treatment of Cancer (EORTC) is conducting a study evaluating the use of one and three years of maintenance therapy (study 30962).

\section{EFFICACY}

The success of BCG as a treatment depends on it being used appropriately. It is employed for three reasons: (1) to treat CIS 
Table 1 Summary of results for CIS and papillary tumours treated with BCG

\begin{tabular}{|c|c|c|c|c|}
\hline Disease & Study & No of patients & Initial response rate (\%) & Median follow up (months) \\
\hline CIS, primary & Akaza et al ${ }^{15}$ & 32 & 84.4 & 20 \\
\hline $\mathrm{CIS}$, primary & Harland et al ${ }^{16}$ & 53 & 53 & 32 \\
\hline $\mathrm{CIS}$, primary & Merz et $a l^{17}$ & 25 & 88 & 44 \\
\hline $\mathrm{CIS}$, secondary & Merz et all & 25 & 78 & 40 \\
\hline Papillary & Akaza et $a l^{15}$ & 125 & 66.4 & 42 \\
\hline
\end{tabular}

or occasionally residual papillary tumours; (2) to reduce the number and frequency of recurrent tumours; and (3) to prevent disease progression (although this remains a controversial point, on which there is no consensus view).

\section{(1) Treatment of CIS or residual papillary tumours Treatment of $\mathrm{CIS}$}

BCG is first line treatment for CIS. It has been shown by Akaza et al in 1995 that with BCG $84.4 \%$ of his 32 cases with CIS showed a complete response..$^{15}$ These results were better than the previously reported figures by Harland et al, which revealed a complete response in only $53 \%$ of their 53 patients. ${ }^{16}$ Although it should be noted that the Harland group used a lower dose of BCG.

Merz et al, in 1995 looked at 115 patients with CIS, 25 of these had primary CIS (not associated with a bladder cancer) and 90 had secondary CIS (this is CIS associated with a bladder cancer). ${ }^{17}$ They found that $88 \%$ of those with primary CIS had responded completely, with negative cytology and cystoscopic findings (median follow up period time of 44 months). Of those with secondary CIS, $78 \%$ had a complete response after treatment with BCG (median follow up time of 40 months). These results are summarised in table 1.

\section{Treatment of papillary tumours}

As an indication for BCG treatment, this is less common and is usually utilised when endoscopic control is not possible, either because the sheer volume of bladder tumour makes complete resection difficult or because the patient is not medically fit for an operative procedure. Akaza et al used BCG as a primary treatment for 125 cases of Ta or Tl tumours, and they were able to achieve a $66.4 \%$ complete response. ${ }^{15}$ A partial response was achieved in a further $20.8 \%$ of their patients.

\section{(2) Recurrence prophylaxis}

Experience has revealed that the majority of patients with bladder cancer will have recurrences. Obviously, to reduce the number or rate of recurrences of superficial bladder cancer, or to prevent them altogether would be beneficial to both patient and health care system. To date there have been many trials comparing the various intravesical therapies, including BCG, which are available for superficial bladder cancer, but direct comparisons between these trials is difficult due to the differing administration regimens and dosages used.

Lamm et al in 1992 reviewed the long term results of intravesical therapies for superficial bladder cancer. ${ }^{18}$ They found that four out of five prospective controlled comparisons of BCG with surgery alone showed a significant reduction of tumour recurrence with BCG. The figures recorded showed an overall tumour recurrence rate of $75 \%$ for the control group and a $31 \%$ recurrence rate for the group treated with BCG. They also reviewed 20 controlled chemotherapy studies and only 11 of them achieved a statistically significant reduction in recurrences. The average recurrence rate for controls was $58 \%$ compared with a recurrence rate of $41 \%$ for those treated with intravesical chemotherapy. Lamm reported that chemotherapy reduced the number of recurrences in the first five years, but after this time period the incidence of recurrence was the same as that of the surgery group. This differs from the BCG group as their incidence of recurrence remained reduced.

Several studies have compared BCG with intravesical chemotherapy. One of these was a study carried out by Lundholm in 1996 when he randomised 261 patients with superficial bladder cancer to receive either BCG or mitomycin C. ${ }^{19}$ After a median follow up period of 39 months, $49 \%$ of those who had been given BCG were disease free compared with only $34 \%$ of the mitomycin C group, these results were statistically significantly different $(\mathrm{p}<0.03)$. Those who did have tumour recurrence had a decreased number of recurrences and a significantly longer time to recurrence if they had received BCG when compared with those who had received mitomycin C. The Southwest Oncology Group (SWOG) study also compared mitomycin C with BCG but as there were significantly fewer recurrences in the BCG group compared to the mitomycin $C$ group $(p=0.006)$ the study was terminated early. ${ }^{20}$ They also noted that the time to recurrence in the BCG group was longer: 36 months compared with 20 months as in the mitomycin $\mathrm{C}$ group.

This favourability of BCG compared with mitomycin C was not demonstrated in all studies. Rubben et al in 1990 found that mitomycin C and BCG had only a 7\% advantage over their controls. ${ }^{21}$ In this study a low recurrence rate of $42 \%$ in the controls may explained the lack of significant difference between the treatments. Vegt et al reported that treatment with BCG and mitomycin $C$ were equally effective. ${ }^{22}$ This lack of difference between the two treatment groups could be explained by the fact that large numbers of the study group had low grade Ta lesions which may have hidden the benefit that BCG would have given over the mitomycin $\mathrm{C}$ to the patients with high grade lesions. The results from these studies are summarised in table 2 .

\section{(3) Progression}

Herr et al compared transurethral resection and BCG with transurethral resection alone. ${ }^{23}$ He reported that $95 \%$ of those treated with transurethral resection underwent disease progression, and 53\% of those treated with transurethral resection and BCG progressed. Muscle invasive and/or metastatic disease occurred with equal frequency between the two groups, but was significantly delayed by treatment with BCG $(p=0.012)$. A greater proportion $(42 \%)$ of the control group underwent cystectomy compared with $26 \%$ of the group who received BCG and transurethral resection; these results were statistically significantly different $(p=0.017)$. They also reported that the time to operation (cystectomy) was shorter in the control group-that is, a mean of eight months compared with a mean of 24 months in the BCG group. An overall mortality of $32 \%$ was recorded in the control group compared with the lower mortality figure of $14 \%$ in the BCG group $(\mathrm{p}=0.032)$.

Lamm et al compared doxorubicin with BCG and a progression rate of $37 \%$ for the doxorubicin group and a progression rate of $15 \%$ for the BCG was recorded $(\mathrm{p}=0.015) .{ }^{24}$ Pagano et al mirrored these results in a further study. ${ }^{25}$

BCG was found to be superior as a treatment for superficial bladder cancer by Martinez-Piniero et al when they compared 
Table 2 Summary of outcome of several studies of intravesical therapy for the recurrence prophylaxis of superficial bladder cancer

\begin{tabular}{|c|c|c|c|c|}
\hline Study & $\begin{array}{l}\text { No of } \\
\text { patients }\end{array}$ & Comparison & Outcome of study & Comment \\
\hline Lamm $^{18}$ & 3166 & $\begin{array}{l}\text { Thiotepa, mitomycin C, } \\
\text { doxorubicin, epirubicin }\end{array}$ & BCG was better & $\begin{array}{l}\text { Large review of } 20 \text { chemotherapy, and } 5 \text { BCG } \\
\text { papers }\end{array}$ \\
\hline Lundholm et $a l^{19}$ & 261 & Mitomycin C, BCG & BCG was better & 2 year treatment in both groups \\
\hline SWOG ${ }^{20}$ & 377 & Mitomycin C, BCG & BCG was better & Terminated early \\
\hline Rubben et $a^{21}$ & 77 & BCG, control & No difference & Controls had a low recurrence rate \\
\hline Vegt et a ${ }^{22}$ & 437 & $\begin{array}{l}\text { BCG (RIVM strain), BCG (Tice } \\
\text { strain), mitomycin C }\end{array}$ & $\begin{array}{l}\text { BCG-RIVM and mitomycin C } \\
\text { were equal }\end{array}$ & $\begin{array}{l}6 \text { weeks of treatment with BCG and } 6 \text { months } \\
\text { treatment with mitomycin C. Large number of low } \\
\text { grade Ta tumours }\end{array}$ \\
\hline
\end{tabular}

it to doxorubicin and thiotepa in a randomised prospective study of 202 patients over 31.3 months. ${ }^{26}$ A statistically significant difference was noted in the progression rates, a $1.5 \%$ rate was noted in the BCG group compared with $3.6 \%$ in the thiotepa group and $7.5 \%$ in the doxorubicin group.

With respect to progression, when BCG is compared with mitomycin $\mathrm{C}$ there appears to be less of a difference. Lundholm et al in a randomised prospective study of 261 patients (median follow up 39 months) compared long term instillations of mitomycin C and BCG. ${ }^{19}$ They reported similar rates of tumour progression (13\%) in the two groups. Again, similar progression rates were also found by Krege et al in a randomised multicentre trial on adjuvant therapy in patients with superficial bladder cancer. ${ }^{27}$ They compared (1) transurethral resection alone with (2) transurethral resection and mitomycin C with (3) transurethral resection and BCG. The mitomycin $\mathrm{C}$ group received their treatment for one year on an alternate week basis and for the second year on a monthly basis, and the BCG group received their treatment weekly for six weeks and after this monthly for four months. The progression rates were surprisingly $4.2 \%$ in all three groups. This was felt to be because the population being studied contained an abundance of patients with low grade Ta lesions which may therefore have masked the benefits in terms of tumour progression rates which the groups treated with BCG and mitomycin $C$ would have enjoyed over the group treated with transurethral resection alone.

\section{SIDE EFFECTS}

Side effects are either BCG specific or non-specific. The non-specific risks relate to the procedure of retrograde urethral catheterisation before BCG instillation. The prevention of these non-specific side effects rely on (1) the passage of a urethral catheter in an atraumatic manner under aseptic conditions and (2) a sterile urine culture before catheterisation.

BCG specific side effects are common. The most common side effects as described by Rischmann et al are abacterial cystitis and dysuria (80\%), haematuria $(40 \%)$, and a low grade pyrexia $(30 \%) .{ }^{28}$ These side effects usually settle within a 48 hour period and require little more than paracetamol in way of treatment. In these cases BCG treatment can continue but should the side effects become more troublesome then one can consider increasing the time period between the treatments or reducing the treatment dose. These side effects mimic a bacterial urinary tract infection and should empirical antibiotics be felt necessary then as described by Durek et al in 1999, ${ }^{29}$ quinolones should be avoided. Durek reported that quinolones have some action against live BCG and therefore there is a potential to reduce the antitumour effect of the BCG immunotherapy.

Granulomatous prostatitis is noted in $1 \%$ of patients, ${ }^{28}$ although it is felt that this is more common but asymptomatic. Symptomatic patients require a three month course of rifampicin and isonazid. Similar treatment is required for epididymitis which occurs in $0.2 \%$. It has been reported that granulomas can occur anywhere throughout the body including the liver, lungs, and kidneys. The latter may represent either a local or haematogenous spread. In this instance BCG treatment should be stopped and triple therapy should be started for a period of three to six months.

In $1 \%$ of patients, allergic reactions, including arthralgia and skin rashes have been reported. Treatment is symptomatic only and BCG is usually stopped. The question of restarting BCG treatment depends on a further risk benefit analysis. If it is decided to give further treatment then the patient needs to be monitored in the first few hours after restarting BCG treatment in case of a more severe reaction.

Contraindications to BCG are an impaired immune response irrespective of whether this impairment is congenital in origin or caused by disease, drugs, or other therapy. It is also contraindicated in pregnancy and lactation and in people with positive HIV serology.

Generalised BCG "itis" is by far the most severe complication of the treatment. A systemic BCG reaction is a rare, systemic granulomatous illness, which may occur subsequent to exposure to BCG. Its aetiology is believed to be either septicaemic, immunoallergenic, or a combination of the two. A systemic BCG reaction is defined as the presence of any of the following signs, if no other aetiologies for such signs are detectable: fever $>39.5^{\circ} \mathrm{C}$ for $>12$ hours; fever of $>38.5^{\circ} \mathrm{C}$ for $>48$ hours; pneumonitis; hepatitis; other organ dysfunction outside of the genitourinary tract with granulomatous inflammation on biopsy; or the classical signs of sepsis, including circulatory collapse, acute respiratory distress, and disseminated intravascular coagulation. ${ }^{30}$ A systemic BCG reaction is much more likely to occur if BCG is administered within one week of either transurethral resection or traumatic bladder catheterisation that was associated with haematuria. Treatment of this requires transfer to an intensive care unit where adequate resuscitation, tuberculosis triple therapy, high dose steroids, and antibiotics with action against Gram negative organisms are given.

\section{MECHANISM OF ACTION}

The exact mechanism of action of intravesical BCG is not completely understood. It is known that an intact immune system is important for the antitumour activities of BCG-this was first demonstrated by Ratliff et al in 1987. ${ }^{31}$ They demonstrated that athymic mice were unable to mount an antitumour response.

Before the immune system is invoked certain other events must take place first. Ratliff et al in 1988 suggested that BCG attached to the bladder surfaces coated with the extracellular matrix protein, fibronectin. ${ }^{32}$ After the adherence of BCG it is believed that it is then endocytosed ${ }^{33}$; this point does not have universal acceptance. After these events a massive cellular infiltration is seen; this local inflammatory reaction in the 
bladder mucosa is characterised by large numbers of $\mathrm{T}$ cells both CD4, CD8, and macrophages. The importance of these were demonstrated again by Ratliff et al who showed that a depletion of these $\mathrm{T}$ cell groups in mice decreased the BCG mediated response. ${ }^{34}$

Jackson and James in 1994, described the antitumour mechanism of BCG by suggesting that there was an immune response and a tumour response. ${ }^{35}$ They reported that the immune response consisted of an inflammatory infiltrate and the secretion of cytokines. They described CD8 T cells killing via the induction of apotosis or necrosis, most likely after recognition of intracellular adhesion molecule (ICAM) and major histocompatibility complex (MHC) II in target cells. The contribution of CD4 T cells is also marked by the secretion of cytokines leading to the maturation of cytotoxic T cells or possibly more specific BCG activated killer cells (BAK). These BAK cells are capable of differentiating between normal and tumour cells, but their exact origin is not known. Interestingly, only viable BCG organisms can induce BAK cell activity, which may explain why live (viable) attenuated BCG is necessary for successful intravesical BCG therapy.

The tumour response relates mainly to a change in the phenotype of tumour cells secondary to cytokine activity, especially that of gamma interferon. The first observations of changes in the phenotype of tumour cells was reported by Prescott et al in 1989, when they reported the induction of MHC II molecules after treatment with BCG. ${ }^{36}$ This was followed by the later discovery that ICAM 1 is also induced after BCG. ${ }^{37}$ There is therefore, an increased expression of ICAM 1 and MHC II after BCG treatment. The ability of activated lymphocytes to conjugate with tumour cells largely depends on the expression of ICAM by the tumour. After the process of conjugation the lymphocytes deliver lethal "hits" to the tumour cell, then detach themselves, and are then ready to kill once more. ${ }^{35} \mathrm{MHC}$ II are involved in antigen presentation. Antigens could be presented to CD4 T cells in association with MHC II expressed by macrophages, lymphocytes, or indeed tumour cells. It has been demonstrated by Lattime et al in 1992 that bladder tumour cells, in a murine model, could present BCG derived antigens to CD4 $\mathrm{T}$ cells isolated from regional lymph nodes. ${ }^{38}$

In addition to the above there is also some evidence that BCG also affects growth rates of tumour cell lines. Bohle et al in 1993 carried out initial studies, which showed that BCG does not exert a direct cytotoxic effect against bladder cancer cells, ${ }^{39}$ but later studies have suggested otherwise. In 1994, Jackson et al, found that BCG organisms markedly decreased the capacity of tumour cell lines to proliferate. ${ }^{40}$

These results coupled with their earlier work led Jackson et al to postulate a two stage mechanism for the best clinical response to BCG treatment: (1) the tumour system and (2) the immune system. They felt that the combined activation of the immune system and a response of the tumour system is necessary for a complete clinical response to intravesical BCG. ${ }^{35}$

\section{FUTURE WORK WITH BCG}

Present research is aimed at attempting to understand the exact mechanism of action of BCG, and towards improving its efficacy, and reducing its side effects, as its present side effect profile prevents its more widespread use. It is hoped that with improved patient selection, adopting lower dose regimens of BCG, and using maintenance therapy, that the results with BCG will improve. There are trials presently being conducted investigating these options.

\section{Authors' affiliations \\ J-P Meyer, D A Gillatt, Bristol Urological Institute, Southmead Hospital, Bristol, UK}

R Persad, Bristol Royal Infirmary, Bristol, UK

\section{Box 3: Summary}

- BCG is a form of intravesical immunotherapy.

- BCG is used in CIS and high grade superficial bladder cancer.

- BCG is given as a course of intravesical treatments, once a week for six weeks.

- BCG can be given as maintenance therapy, although this is presently the subject of a EORTC study.

- The mechanism of action of BCG is not fully understood.

- Future research is aimed at improving efficacy and reducing side effects.

\section{REFERENCES}

1 Lutzeyer W, Rubben $\mathrm{H}$, Dahm H. Prognostic parameters in superficial bladder cancer: an analysis of 315 cases. J Urol 1982;127:250-2.

2 Oosterlinck W. The management of superficial bladder cancer. BJU Int 2001;87:135-40

3 Millan-Rodriguez F, Chechile-Toniolo G, Salvador-Bayarri J, et al. Multivariate analysis of the prognostic factors or primary superficial bladder cancer. J Urol 2000;163:73-8.

4 Zieger $\mathrm{K}$, Wolf $\mathrm{H}$, Olsen PR, et al. Long-term survival of patients with bladder tumours: the significance of risk factors. $\mathrm{Br} J$ Urol 1998;82:667-72.

5 Zieger $\mathrm{K}$, Wolf $\mathrm{H}$, Olsen PR, et al. Long-term follow-up of noninvasive bladder tumours (stage Ta): recurrence and progression. BJU Int 2000;85:824-8.

6 Guerin C. Early history of BCG. In: Rosenthal SR, ed. BCG vaccination against tuberculosis. Boston: Little Brown, 1957: 48-53.

7 Pearl R. Cancer and tuberculosis 1929:9:97-159.

8 Mathe G, Amiel JL, Schwarzenburg L, et al. Active immunotherapy for acute lymphoblastic leukaemia. Lancet 1969;i:697-9.

9 Coe JE, Feldman JD. Extracutaneous delayed hypersensitivity particularly in guinea pig bladder. Immunology 1966;10:127-36.

10 Morales A, Eidinger D, Bruce AW. Intracavity BCG in the treatment of superficial bladder tumours. J Urol 1976;116:180-3.

11 Lamm DL, Thor DE, Harris SC, et al. BCG immunotherapy of superficial bladder cancer. J Urol 1980;124:38-40.

12 Bretton PR, Herr HW, Kimmel M, et al. The response of patients with superficial bladder cancer to a second course of intravesical BCG. J Uro 1990;143:710-13

13 Catalona WJ, Hudson MA, Gillen DP, et al. Risks and benefits of repeated courses of intravesical BCG for superficial bladder cancer. J Urol 1987; 137:220-4.

14 Lamm DL, Blumenstein BA, Crismon JD, et al. Maintenance BCG immunotherapy for recurrent TA, T1 and CIS TCC of the bladder: a randomised Southwest Oncology Group Study. J Urol 2000; 163: 1 1 124-9.

15 Akaza H, Hinotsu S, Aso Y, et al. BCG treatment of existing papillary cancer and carcinoma in situ of the bladder. Cancer 1995:75:552-9.

16 Harland SJ, Charing CR, Highman W, et al. Outcome in carcinoma in situ of bladder treated with intravesical BCG. Br J Urol 1992;70:271-5.

17 Merz VW, Marth D, Kraft R, et al. Analysis of early failures after intravesical installation therapy with BCG for carcinoma in situ of the bladder. Br J Urol 1995:75:180-4.

18 Lamm DL. Long term results of intravesical therapy for superficial bladder cancer. Urol Clin North Am 1992;19:573-80.

19 Lundholm C, Norten BJ, Eckman P, et al. A randomised prospective study comparing long term intravesical installations of $M M C$ and BCG in patients with superficial bladder cancer. J Urol 1996:156:372-6.

20 Lamm DL, Crawford ED, Blumenstein B, et al. SWOG 8795. A randomised comparison of BCG and MMC prophylaxis in stage Ta \& $T$ transitional cell carcinoma of the bladder. J Urol 1993;149:282A (abstract 275).

21 Rubben H, Graf-Doberstein C, Ostwald R, et al. Prospective randomised study of adjuvant therapy after complete resection of superficial bladder cancer: MMC vs BCG vs TUR alone. Immunotherapy of urological tumours. Edinburgh: Churchill Livingstone, 1990: 27-36.

22 Vegt PDJ, Witjes A, Witjes WH, et al. A randomised study of intravesical mitomycin C, BCG-tice and BCG-rivm treatment in pTa, pT 1 papillary carcinoma and CIS of the bladder. J Urol 1995;153:929-39.

23 Herr HW, Laudone VP, Badalament RA, et al. BCG therapy alters the progression of superficial bladder cancer. J Clin Oncol 1988;6:1450-5.

24 Lamm DL, Blummenstein BA, Crawford ED, et al. A randomised trial of intravesical doxorubicin and immunotherapy with BCG of transitional cell carcinoma of the bladder. N Engl J Med 1991;325:1205-9.

25 Pagano F, Bassi P, Milani C, et al. A low dose of BCG regimen in superficial bladder cancer. Is it effective? J Urol 1991;146:32-5

26 Martinez-Pineiro JA, Jimenez Leon J, Martinez-Pineiro L, et al. BCG versus doxorubicin versus thiotepa: a randomised prospective study in 202 patients with superficial bladder cancer. J Urol 1990;143: 502-6. 
27 Krege S, Otto $\mathrm{T}$, Rubben $\mathrm{H}$, et al. Final report on a randomised multicentre trial on adjuvant therapy in superficial bladder cancer. TUR only vs TUR \& MMC vs TUR \& BCG. J Urol 1996;155:494A (abstract 734).

28 Rischmann P, Desgrandchamps F, Malavaud B, et al. BCG intravesical installations: recommendations for side-effects management. Eur Urol 2000;37(suppl 1):33-6

29 Durek C, Rusch-Gerdes S, Jocham D, et al. Interference of modern antibacterials with bacillus Calmette-Guérin viability. J Urol 1999:163:1959-62.

30 Lamm DL. Incidence and treatment of complications of bacillus Calmette Guérin intravesical therapy in superficial bladder cancer. J Urol 1992;147:596-600

31 Ratliff TL, Gillen D, Catalona WJ. Requirement of a thymus dependent immune response for BCG mediated antitumour activity. J Urol 1987: 137:155-8.

32 Ratliff TL, Kavoussi LR, Catalona WJ. Role of fibronectin in intravesical BCG therapy for superficial bladder cancer. J Urol 1988;139:410-14.

33 Beich MJ, Carroll S, Ratliff TL. Internalisation of BCG by bladder tumour cells. J Urol 1991;145:1316-24.
34 Ratliff TL, Ritchey JK, Yuan Jl, et al. T-cell subsets required for intravesical BCG immunotherapy for bladder cancer. J Urol 1993; 150:1018-23.

35 Jackson AM, James K. Understanding the most successful immunotherapy for cancer. The Immunologist 1994;2:208-15.

36 Prescott $\mathrm{S}$, James $\mathrm{K}$, Busutill $\mathrm{A}$, et al. HLA-DR expression by high grade superficial bladder cancer treated with BCG. Br J Urol 1989;63:264-9.

37 Jackson AM, Alexandroff AB, Mclntyre M, et al. BCG immunotherapy induces ICAM-1 expression on bladder tumours. J Clin Pathol 1994:47:309-12.

38 Lattime EC, Gomella LG, McCue PA. Murine bladder carcinoma cells present antigen to BCG specific CD4 T cells. Cancer Res 1992;52:4286-90.

39 Bohle A, Thanhauser A, Ulmer AJ, et al. Dissecting the immunobiologica effects of bacillus Calmette-Guérin organisms in vitro: evidence of a distinct BCG activated killer (BAK) cell phenomenon. J Urol 1993;150:1932-7.

40 Jackson AM, Alexandroff AB, Fleming D, et al. Bacillus Calmette-Guérin organisms directly affect the growth of bladder tumour cells. Int J Urol 1994;5:697-703.

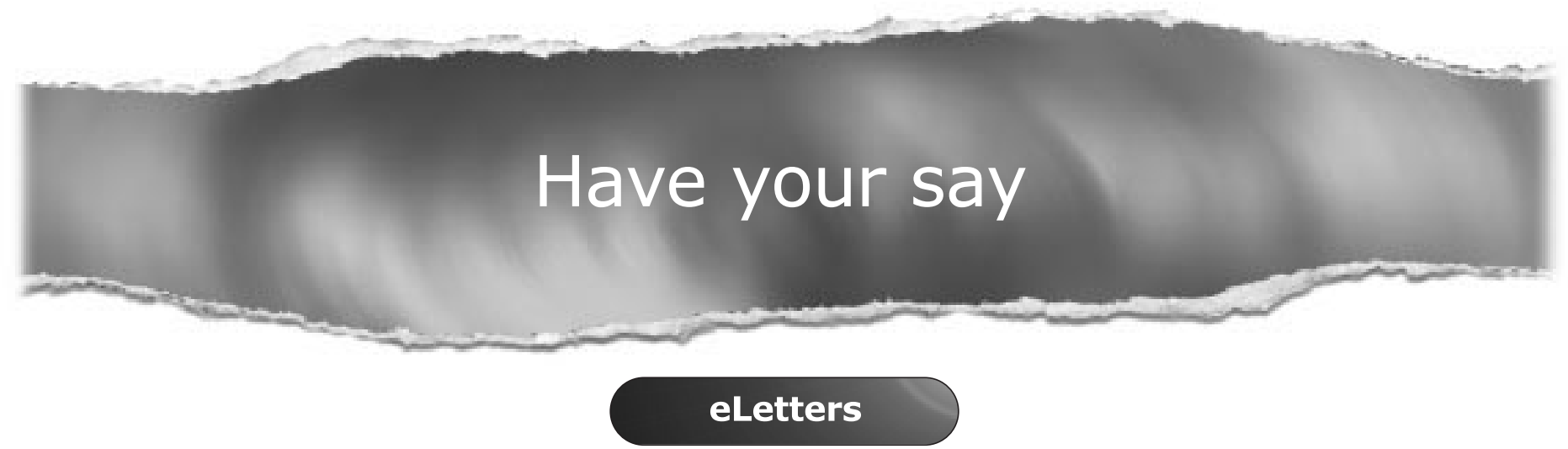

If you wish to comment on any article published in the Postgraduate Medical Journal you can send an eLetter using the eLetters link at the beginning of each article. Your response will be posted on Postgraduate Medical Journal online within a few days of receipt (subject to editorial screening).

\section{www.postgradmedj.com}

